\title{
Pathological myopia-induced antioxidative proteins in the vitreous humor
}

\author{
Qiaoling Wei ${ }^{1,2 \#}$, Ting Zhang ${ }^{1,2 \#}$, Jiawen Fan ${ }^{1,2}$, Rui Jiang ${ }^{1,2}$, Qing Chang ${ }^{1,2}$, Jin Hong ${ }^{3}$, Gezhi Xu ${ }^{1,2}$ \\ ${ }^{1}$ Ophthalmology Department, EYE \& ENT Hospital of Fudan University, Shanghai 200031, China; ${ }^{2}$ Key Laboratory of Visual Impairment and \\ Restoration of Fudan University, Shanghai 200031, China; ${ }^{3}$ Institutes of Biomedical Sciences, Fudan University, Shanghai 200031, China \\ Contributions: (I) Conception and design: Q Wei, T Zhang, G Xu; (II) Administrative support: G Xu; (III) Provision of study materials or patients: \\ All authors; (IV) Collection and assembly of data: All authors; (V) Data analysis and interpretation: Q Wei, T Zhang; (VI) Manuscript writing: All \\ authors; (VII) Final approval of manuscript: All authors. \\ \#These authors contributed equally to this work. \\ Correspondence to: Prof. Gezhi Xu. EYE \& ENT Hospital of Fudan University, No. 83 Fenyang Road, Xuhui District, Shanghai 200031, China. \\ Email: Drxugezhi@163.com.
}

Background: This study aimed to investigate differentially expressed proteins in the vitreous humor (VH) of pathological myopia (PM) and normal eyes.

Methods: VH samples were collected from patients undergoing surgical treatment for rhegmatogenous retinal detachment (RRD), idiopathic epiretinal membrane (ERM), myopic retinoschisis (MRS) or macular hole $(\mathrm{MH})$. A label-free quantitative proteomic analysis was performed to detect the differentially expressed proteins, and expression of three differentially expressed proteins was confirmed by ELISA.

Results: In PM patients (MH-PM, MRS-PM and RRD-PM), the expression of prostaglandin-H2 D-isomerase (PGDS) and glutathione peroxidase 3 (GPX3) was significantly lower than in controls (MH, ERM, and RRD). The versican core protein expression decreased in the PM group. The vitreous concentrations of PGDS and GPX3 in patients with axial length (AL) of 26.5-29.0 mm were higher than in patients with $\mathrm{AL}>29.0 \mathrm{~mm}$ or $\mathrm{AL}<26.5 \mathrm{~mm}$. NRF-2 expression was the lowest in patients with AL $>29.0 \mathrm{~mm}$.

Conclusions: Our study provides new evidence on the molecular changes in the VH of PM patients, and these molecules have the potential to become new targets for the therapy of PM.

Keywords: Pathological myopia (PM); retinal pigment epithelium (RPE); prostaglandin-H2 d-isomerase (PGDS); antioxidative protein

Submitted Oct 28, 2019. Accepted for publication Jan 02, 2020.

doi: $10.21037 /$ atm.2020.01.63

View this article at: http://dx.doi.org/10.21037/atm.2020.01.63

\section{Introduction}

Pathological myopia (PM) is a major cause of irreversible vision impairment due to its complications, such as chorioretinal atrophy, choroidal neovascularization and macular atrophy $(1,2)$, especially in East Asia (3). Even the global prevalence of myopia and high myopia is increasingly at an alarming rate in the past five decades (4), the definition of PM is still controversial (5). In a published meta-analysis, diffuse chorioretinal atrophy or chorioretinal atrophy more severe than diffuse atrophy is included as one of the required signs for the diagnosis of $\mathrm{PM}$ (6).

Older age, higher myopic spherical equivalent refractive error (SRE), longer axial length (AL) as well as posterior staphyloma have been found as an important risk factors for more severe myopic maculopathy $(7,8)$. Posterior staphyloma, which can be detected by ultrasonography $(7,8)$, may cause serious mechanical damage to both retinochoroidal tissues and optic nerve, resulting in myopic maculopathy. With the increasing age and continuous 
extension of the eye axis, scleral staphyloma increases and then the degenerative processes are accelerated in the posterior segment of the eyes, including the thinning of photoreceptor layers and retinal pigment epithelium (RPE), some of which can even disappear completely (9). The irreversible atrophy of the RPE and photoreceptor layers may cause untreatable visual impairment. PM also frequently predisposes patients to other eye diseases such as macular hemorrhage, cataracts, open angle glaucoma, peripapillary atrophy and retinal detachment.

The progressive prolongation of the eyeball AL and increasing age are the main causes of the functional deterioration associated with PM. Oxidative stress has been proposed as a pathophysiological mechanism underlying the pathogenesis of PM (10). In vivo study shows oxidative stress initiates the degenerative process of neural retina (11). However, few studies have focused on the underlying pathophysiological mechanisms of myopic retinopathy, and many issues remain to be resolved. The vitreous humor (VH) is an accessible compartment adjacent to the retina and may therefore represent the microenvironment of the retina. Our previous study showed that matrix metalloproteinase 2 (MMP-2) expression in the $\mathrm{VH}$ of high myopia patients was significantly higher than in controls, supporting the practice that the $\mathrm{VH}$ can be used to investigate the intraocular pathological changes associated with PM (12). Proteomics is a useful approach to investigate the total proteins, which together with a robust statistical analysis can identify the proteins associated with a specific ophthalmological disease $(13,14)$. Therefore, a label-free quantitative proteomic analysis was performed in this study to investigate the differentially expressed proteins in the $\mathrm{VH}$ of $\mathrm{PM}$ and normal eyes.

\section{Methods}

\section{Patients and grouping}

This was a cross-sectional, observational study which was approved by the Institutional Review Board of the EYE \& ENT Hospital of Fudan University. Our study was conducted according to the ethical principles of the Declaration of Helsinki. VH samples were collected from patients who underwent surgical treatment for rhegmatogenous retinal detachment (RRD), idiopathic epiretinal membrane (ERM), myopic retinoschisis (MRS), and macular hole $(\mathrm{MH})$ in the EYE \& ENT Hospital of Fudan University between August 2015 and May 2017.
After the informed consent was obtained, each patient received comprehensive preoperative eye examinations, including slit-lamp examination, indirect ophthalmoscopy, visual acuity test, SRE evaluation, AL (IOLmaster 500, ZEISS), ophthalmic ultrasonography and fundus optical coherence tomography. The clinical characteristics of these patients (such as age, sex, and systemic diseases) were also recorded.

The surgically treated eyes that had SRE less negative than $-6.0 \mathrm{D}$, AL longer than $26.5 \mathrm{~mm}$ and chorioretinal degeneration fundus were included in the PM groups. The signs of chorioretinal degeneration included fundus tessellation, macular atrophy, and diffuse or patchy chorioretinal atrophy, with or without lacquer cracks and Fuchs spot. Meanwhile, the surgically treated eyes that had SRE $>-6.00 \mathrm{D}$ and $\mathrm{AL}<26.5 \mathrm{~mm}$ but had no any chorioretinal degeneration were included in the control group (6). Exclusion criteria were as follows: autoimmune retinal diseases, history of ocular surgery, multiple intravitreal injections, and a history of use of drugs that may affect the VH. The severity of cataract in each patient was graded with the Lens Opacities Classification System, version III (LOCS III) and the myopic degeneration was graded according to previously reported $(6,15)$. Patients were subdivided into six subgroups: $\mathrm{MH}, \mathrm{MH}-\mathrm{PM}$, ERM, MRS-PM, RRD and RRD-PM.

\section{VH collection}

VH samples were collected according to previously reported (16). Briefly, the valve of the vitrector aspiration line was connected to a sterile syringe $(5 \mathrm{~mL})$. Under the closure of the aspiration line, undiluted core VH (500$1,000 \mu \mathrm{L}$ ) was aspirated into the syringe by active vitrector cutting and syringe suction $(25 \mathrm{G}$; Constellation; Alcon Instruments, USA). All VH samples were snap-frozen and then stored at $-80^{\circ} \mathrm{C}$ until assay.

\section{Sample processing}

VH samples were processed according to previously reported (14). First, each VH sample was centrifuged at $4{ }^{\circ} \mathrm{C}$ for $15 \mathrm{~min}$ at $21,000 \mathrm{~g}$ to remove cells and cell debris. Second, the total protein concentration of each $\mathrm{VH}$ sample was determined with the Pierce BCA Protein Assay Kit (Thermo Scientific, MA USA) according to the manufacturer's instructions. Then, $100 \mu \mathrm{L}$ of vitreous sample was collected for further proteomics analysis. 


\section{Liquid chromatography-mass spectrometry (LC-MS)}

Two hundred $\mu \mathrm{g}$ of protein in each sample was used for the LC-MS. The extracted protein was reduced with $10 \mathrm{mM}$ dithiothreitol, alkylated with $20 \mathrm{mM}$ iodoacetamide, and then digested with sequencing-grade modified trypsin (Promega, Madison, WI, USA) in the presence of $1 \mathrm{M}$ urea. The resultant peptides were purified by using the C18 microspin column (Nest Group, Southborough, MA, USA).

\section{Data acquisition}

The data of LC-MS/MS were obtained according to previously reported (16). After separation by nano-highperformance LC (Eksigent Technologies), the peptides were eluted with linear gradient from $5 \%$ to $45 \%$ of buffer B (98\% acetonitrile with $0.1 \%$ formic acid) for $5-70 \mathrm{~min}$ at a flow rate of $300 \mathrm{~nL} / \mathrm{min}$. The electrospray voltage was $2.3 \mathrm{kV}$ for the MS inlet. Under the data-dependent mode, the Triple TOF 4600 MS altered automatically between MS and MS/MS mode. The MS spectra were obtained in the mass range of $350-1,250 \mathrm{~m} / \mathrm{z}$, and the cumulative time of each spectrum was $250 \mathrm{~ms}$. The tandem MS scans were obtained by rolling the collision energy setting in the mass range of $100-1,250 \mathrm{~m} / \mathrm{z}$. The 20 most intense precursors were selected to fragment per cycle, and the dynamic exclusion time was $9 \mathrm{~s}$.

\section{Label-free quantification}

The MaxQuant software (version 1.5.3.30) was applied to process all raw data. The Andromeda search engine was used for protein identification. The enzymatic cleavage was done with trypsin/P, and up to two missed cleavages were allowed. The mass tolerance of the fragment ions was $20 \mathrm{ppm}$. Cysteine carbamidomethylation was selected as fixed modification while protein $\mathrm{N}$-terminal acetylation and methionine oxidation as variable modifications. The false discovery rate was set to 0.01 for both peptide and protein identification. The iBAQ values calculated with MaxQuant were used to quantify the expression of proteins in the $\mathrm{VH}$.

\section{Confirmation by enzyme-linked immunosorbent assay (ELISA)}

The expression of selected differentially expressed proteins in the VH was further confirmed by using commercially available ELISA kits according to the manufacturers' instructions. The prostaglandin D synthase (lipocalintype; human) ELISA Kit (No. 10007684; Cayman Chemical, Cambridge, MA, USA), GPX3 (human) ELISA Kit (AdipoGEN Life Sciences, Liestal, Switzerland), and human NRF2 ELISA kit (RayBiotech, Norcross, GA, USA) were used in the present study.

\section{Statistical analysis}

Statistical analysis was performed using Perseus software (Max Planck Institute of Biochemistry). iBAQ values of six subgroups (MH, MH-PM, ERM, MRS-PM, RRD and RRD-PM) calculated with MaxQuant were used to compare the signal intensity between PM patients and controls. Student's $t$-test (two-tailed) was performed to compare the age, sex, SRE, AL, cataract grade, and signal intensity of differentially expressed proteins between PM patients and controls. One-way analysis of variance (two-tailed) was applied to compare the SRE, AL and protein concentrations among groups with different $\mathrm{AL}$ ranges. A value of $\mathrm{P}<0.05$ was considered statistically significant.

\section{Results}

\section{Characteristics of patients}

A total of $132 \mathrm{VH}$ samples were collected from 132 patients: 22 from $M H$ patients, 22 from $M H+P M$ patients, 22 from MRS-PM patients and 22 from ERM patients, 22 from RRD patients, 22 from RRD + PM patients. The patients' characteristics are shown in Tables 1,2 and supplementary Table 1 (available online: http://cdn.amegroups.cn/static/ application/e85cdd6984f2a1ca489da4f39f345520/10.21037a tm.2020.01.63-1.pdf).

\section{Differentially expressed proteins in the eyes with and without PM}

In PM patients (MH-PM, MRS-PM, and RRD-PM), the expression of prostaglandin-H2 D-isomerase (PGDS) and glutathione peroxidase 3 (GPX3) was significantly lower than in controls (MH, ERM, and RRD). Both PGDS and GPX3 are responsible for the scavenging of reactive oxygen species (ROS) (17). The expression of interphotoreceptor matrix proteoglycan 1 , a component of basic macromolecular scaffold for the insoluble interphotoreceptor matrix, reduced in the PM patients (18). Versican core protein, a protein related to intercellular 
Table 1 Characteristics of patients included in our study



Data are expressed as the number of patients, number (\%) of patients, or mean (range). *, $t$-test. PM, pathological myopia; VH, vitreous humor; $\mathrm{MH}$, macular hole; RRD, rhegmatogenous retinal detachment; MRS, myopic retinoschisis; ERM, epiretinal membrane; SRE, spherical equivalent refractive error; D, diopters; $A L$, axial length.

Table 2 Characteristics of PM patients based on fundus examination and ultrasonographic findings

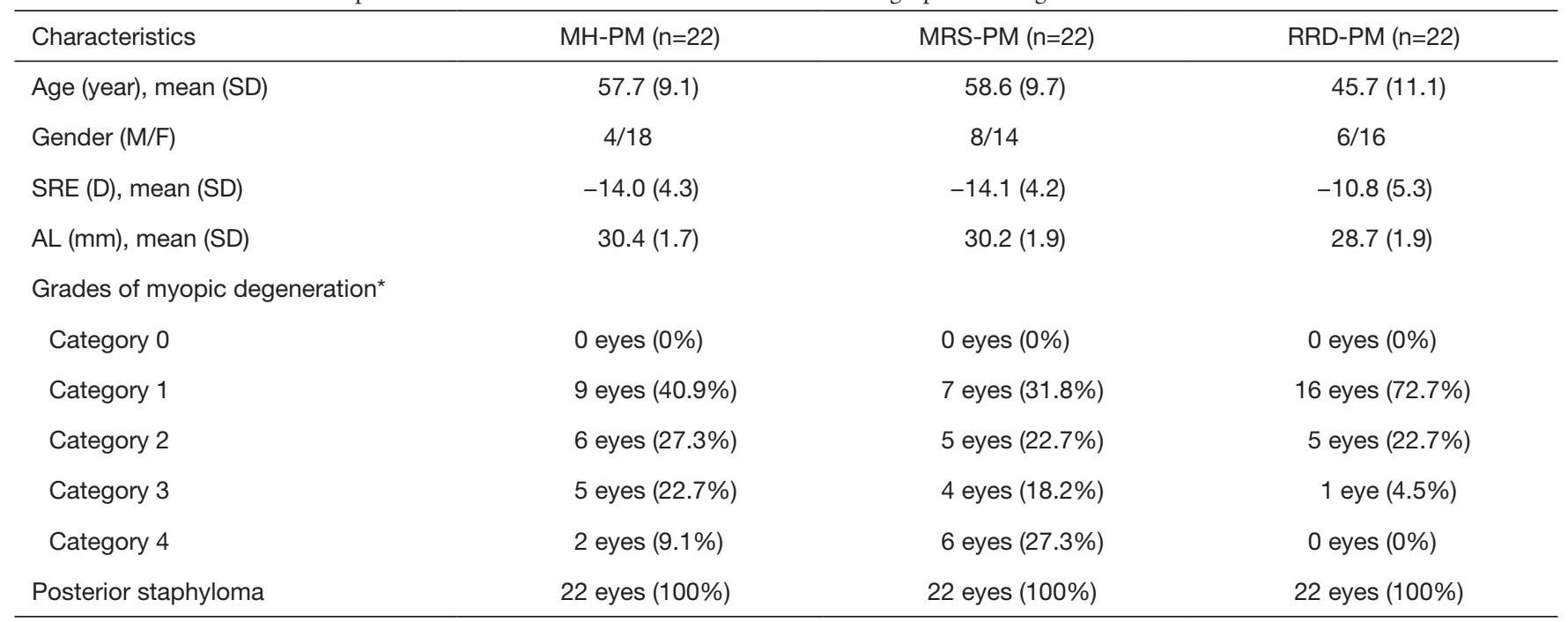

*, grade of myopic degeneration: 0, no macular lesions; 1 , tessellated fundus; 2, diffuse chorioretinal atrophy; 3, patchy chorioretinal atrophy; 4, macular atrophy. PM, pathological myopia; MH-PM, macular hole with pathological myopia; MRS-PM, myopic retinoschisis with pathological myopia; RRD-PM, rhegmatogenous retinal detachment with pathological myopia; SD, standard deviation; SRE, spherical equivalent refractive error; $D$, diopters; $A L$, axial length.

signaling process and cells connecting to the extracellular matrix, decreased in the PM patients [supplementary Table 2 (available online: http://cdn.amegroups.cn/static/applic ation/725d40393af83b66d6a $7195 \mathrm{f} 7728193 \mathrm{~b} / 10.21037 \mathrm{a}$ tm.2020.01.63-2.pdf), Figure 1A,B,C,D]. Volcano plots were constructed to determine the fold change in the expression of each protein $[\mathrm{X}$-axis $=\log 2$ (fold change: $\mathrm{PM}$ patients/controls), Y-axis $=-\log 10(\mathrm{P}$ value $)]($ Figure $2 A)$. Hierarchical clustering analysis was done for the 13 differentially expressed proteins as shown in Figure $2 B$.

\section{Confirmation of selected differentially expressed proteins}

The patients were divided into three groups according to their AL and SER: nonmyopic patients with AL $<26.5 \mathrm{~mm}$, myopic patients with $\mathrm{AL} \geq 26.5 \mathrm{~mm}$ but $<29.0 \mathrm{~mm}$, and myopic patients with $\mathrm{AL} \geq 29.0 \mathrm{~mm}$ [detailed in supplementary Table 3 (available online: http://cdn. amegroups.cn/static/application/f3be356d506aa195e60 c46a9cc81fcf1/10.21037atm.2020.01.63-3.pdf)]. ELISA was employed to confirm the expression of selected differentially expressed proteins. Eighteen $\mathrm{VH}$ samples 



Figure 1 Comparison of PM patients and controls. (A) Signal intensity of prostaglandin-H2 d-isomerase; (B) signal intensity of glutathione peroxidase 3; (C) signal intensity of interphotoreceptor matrix proteoglycan 1; (D) signal intensity of versican core protein; Signal intensities were determined by label-free quantitative proteomics analysis. Student's $t$-test was used to compare the signal intensities of differentially expressed proteins between 2 groups. PM, pathological myopia.

randomly selected from each subgroup were used to detect the vitreous PGDS concentration. Unfortunately, most of samples had run out, and thus, the concentrations of GPX3 and NRF-2 were detected in the remaining VH samples. ELISA results are shown in Table 3 and supplementary Table 3 (available online: http://cdn.amegroups.cn/static/ application/f3be356d506aa195e60c46a9cc81fcf1/10.21 037 atm.2020.01.63-3.pdf). The vitreous concentrations of PGDS and GPX3 were higher in patients with AL of $26.5-29.0 \mathrm{~mm}$ than in those with $\mathrm{AL}>29.0 \mathrm{~mm}$ or $\mathrm{AL}<26.5 \mathrm{~mm}$. NRF-2, and they were the lowest in patients with AL $>29.0 \mathrm{~mm}$.

\section{Discussion}

A recent study shows a remarkable increase in the prevalence of high and very high myopia among high school students in China (19). With the help of glasses or contact lenses as well as corneal or intraocular surgery, the optical defect of myopia can be compensated. However, no strategies have been developed to effectively prevent and treat the impaired vision caused by PM. Currently, the factors related to the occurrence and development of myopia are still unknown. The lack of an animal model that accurately simulates human pathological myopic 




Figure 2 Differentially expressed proteins in the VH of PM patients and controls (label free quantitative proteomics analysis). (A) Volcano plot represents the differentially expressed protein. The $-\log 10$ (P value) is plotted against the difference of the means of two groups (PM and controls). Dots above the non-axial horizontal line are significantly differentially expressed proteins. Thirteen proteins were found to be significantly $(\mathrm{P}<0.002)$ downregulated in the PM samples (green plots), while none was upregulated (red plots), and the proteins with unchanged expression were marked with dark dots. (B) Hierarchical clustering analysis of 13 differentially expressed proteins between PM patients and controls. Protein names are listed on the right, group names are indicated at the top while the subgroups are listed at the bottom. The color key on the left of the heatmap indicates the expression level (red = up-regulation, blue = downregulation). VH, vitreous humor; PM, pathological myopia; ERM, epiretinal membrane; MH, macular hole; RRD, rhegmatogenous retinal detachment; MRS, myopic retinoschisis.

Table 3 Expression of PGDS, GPX3 and Nrf-2 in the VH (ELISA)

\begin{tabular}{|c|c|c|c|c|}
\hline Variables & High PM group & PM group & Control group & $\mathrm{P}^{*}$ \\
\hline Axial length (mm) & $31.1(1.2)$ & $27.6(0.9)$ & $23.7(1.0)$ & $<0.001$ \\
\hline PGDS (ng/mL) & $2,071.6(326.6)$ & $2,702.4(704.4)$ & $2,179.2(271.6)$ & 0.001 \\
\hline GPX3 (ng/mL) & $2,659.3(1,277.5)$ & $5,372.4(1,694)$ & $4,209.1(1,819.8)$ & 0.007 \\
\hline
\end{tabular}

Refractive diopter is expressed as the mean (range) and the remaining data are expressed as the mean (standard deviation). *, oneway analysis of variance. Control group, patients with axial length $<26.5 \mathrm{~mm}$; PM group, PM patients with $26.5 \mathrm{~mm}<$ axial length < 29.0 mm; high PM Group, PM patients with axial length $>29.0$ mm; VH, vitreous humor; PM, pathological myopia; PGDS, prostaglandin-H2 D-isomerase; GPX3, glutathione peroxidase 3; Nrf-2, nuclear factor erythroid 2-related factor. 
retinopathy and the difficulties in obtaining human retinal specimens with various pathological changes significantly limit the investigation of pathogenic mechanisms underlying the pathogenesis PM. The changes in the $\mathrm{VH}$ may reflect the microenvironment changes in case of retinopathy. Therefore, label-free quantitative proteomics was employed to analyze the differentially expressed proteins in the $\mathrm{VH}$ of patients with and without PM.

The downregulation of interphotoreceptor matrix proteoglycan 1 in PM patients suggests the vitreous structural change in the PM. PGDS, which catalyzes the production prostaglandin D2 (PGD2), is involved in transporting the lipophilic substances in the interphotoreceptor matrix, modulating the immune and inflammatory responses and inducing apoptosis and neuroprotection (20-23). There is evidence showing that retinopathy may influence the vitreous concentration of L-PGDS, and the L-PGDS expression has been found to increase in the human subretinal fluid during retinal detachment and in the $\mathrm{VH}$ during proliferative diabetic retinopathy (24-26). Furthermore, in vitro experiments have indicated that L-PGDS can potentially prevent the oxidative stress and apoptosis-related neurodegenerative diseases (27). The label-free proteomics and ELISA in the present study indicated that PGDS was differentially expressed in the $\mathrm{VH}$ of patients with different ALs (Table 3). Because RPE is the main source of intraocular PGDS, we speculate that the increased PGDS in patients with mild global extension (when the RPE functions normally) may exert protective effects by attenuating intraocular oxidative stress and inhibiting neuronal apoptosis; when the atrophy of the RPE reaches a threshold, the synthesis of PGDS decreases.

The ELISA showed that NRF-2 decreased as AL increased (Table 3). NRF-2, a major anti-oxidative protein, is involved in the pathogenesis of many diseases and provides a mechanism for cellular autonomic protection (28). It has been shown the pivotal role of NRF-2 in reducing inflammation and promoting PGDS expression, because the positive feedback by which PGD2 induces L-PGDS expression is the key mechanism by which NRF-2 regulates inflammation $(29,30)$. We hypothesize that the decrease in the expression of PGDS and NRF-2 may be one of molecular mechanisms underlying the pathogenesis of pathological retinopathy.

As a member of the glutathione peroxidase family, GPX3 catalyzes the reduction of organic hydroperoxides and hydrogen peroxide $\left(\mathrm{H}_{2} \mathrm{O}_{2}\right)$ to protect cells from oxidative damage $(17,31)$. The change in the GPX3 expression in case of PM is similar to that of PGDS: it increased in patients with mild global extension $(26.5<\mathrm{AL}<29.0 \mathrm{~mm})$, but decreased in those with $\mathrm{AL}>29.0 \mathrm{~mm}$.

\section{Conclusions}

The expression of L-PGDS, NRF-1 and GXP3, all of which have strong antioxidative capability, decreases in the patients who have fundus chorioretinal degeneration with AL longer than $29.0 \mathrm{~mm}$. The downregulation of PGDS, GPX3 and NRF-2 in patients with AL $>29.0 \mathrm{~mm}$ may represent the severe oxidative stress. Our findings suggest that the oxidation/anti-oxidation imbalance is an important mechanism underlying the pathogenesis of PMrelated retinopathy. Our study provides new insights into understanding the molecular changes in the pathogenesis of PM and new targets for the therapy of PM.

However, there were still limitations in the present study. The sample size was small, and only 132 vitreous samples were collected. Furthermore, the volume of each vitreous sample was too small to confirm the concentrations of all the differentially expressed proteins. Moreover, further investigation is needed to investigate the causal relationship between these differentially expression proteins in the $\mathrm{VH}$ and $\mathrm{PM} / \mathrm{PM}$-associated chorioretinal pathology.

\section{Acknowledgments}

Funding: This study was supported by Shanghai Key Laboratory of Visual Impairment and Restoration (No. 12DZ2260500), China National Natural Science Foundation (No. 81770944) and "Key NHC Key Laboratory of Myopia (Fudan University); Laboratory of Myopia, Chinese Academy of Medical Sciences”.

\section{Footnote}

Conflicts of Interest: The authors have no conflicts of interest to declare.

Ethical Statement: The authors are accountable for all aspects of the work in ensuring that questions related to the accuracy or integrity of any part of the work are appropriately investigated and resolved. The study was approved by the ethic committee of EYE \& ENT Hospital of Fudan University (No. 2017-03-01). Written informed consent was obtained from all patients. 
Open Access Statement: This is an Open Access article distributed in accordance with the Creative Commons Attribution-NonCommercial-NoDerivs 4.0 International License (CC BY-NC-ND 4.0), which permits the noncommercial replication and distribution of the article with the strict proviso that no changes or edits are made and the original work is properly cited (including links to both the formal publication through the relevant DOI and the license). See: https://creativecommons.org/licenses/by-nc-nd/4.0/.

\section{References}

1. Foster PJ, Jiang Y. Epidemiology of myopia. Eye (Lond) 2014;28:202-8.

2. Wong TY, Ferreira A, Hughes R, et al. Epidemiology and disease burden of pathologic myopia and myopic choroidal neovascularization: an evidence-based systematic review. Am J Ophthalmol 2014;157:9-25.e12.

3. Chan NS, Teo K, Cheung CM. Epidemiology and Diagnosis of Myopic Choroidal Neovascularization in Asia. Eye Contact Lens 2016;42:48-55.

4. Koh V, Yang A, Saw SM, et al. Differences in prevalence of refractive errors in young Asian males in Singapore between 1996-1997 and 2009-2010. Ophthalmic Epidemiol 2014;21:247-55.

5. Morgan IG, Ohno-Matsui K, Saw SM. Myopia. Lancet 2012;379:1739-48.

6. Ohno-Matsui K, Kawasaki R, Jonas JB, et al. International photographic classification and grading system for myopic maculopathy. Am J Ophthalmol 2015;159:877-83.e7.

7. Steidl SM, Pruett RC. Macular complications associated with posterior staphyloma. Am J Ophthalmol 1997;123:181-7.

8. Xiao O, Guo X, Wang D, et al. Distribution and Severity of Myopic Maculopathy Among Highly Myopic Eyes. Invest Ophthalmol Vis Sci 2018;59:4880-5.

9. Ohno-Matsui K, Lai TY, Lai CC, et al. Updates of pathologic myopia. Prog Retin Eye Res 2016;52:156-87.

10. Francisco BM, Salvador M, Amparo N. Oxidative stress in myopia. Oxid Med Cell Longev 2015;2015:750637.

11. Ham WT Jr, Mueller HA, Ruffolo JJ Jr, et al. Basic mechanisms underlying the production of photochemical lesions in the mammalian retina. Curr Eye Res 1984;3:165-74.

12. Zhuang H, Zhang R, Shu Q, et al. Changes of TGF-beta2, MMP-2, and TIMP-2 levels in the vitreous of patients with high myopia. Graefes Arch Clin Exp Ophthalmol 2014;252:1763-7.
13. Levin Y, Schwarz E, Wang L, et al. Label-free LC-MS/ MS quantitative proteomics for large-scale biomarker discovery in complex samples. J Sep Sci 2007;30:2198-203.

14. Megger DA, Bracht T, Meyer HE, et al. Label-free quantification in clinical proteomics. Biochim Biophys Acta 2013;1834:1581-90.

15. Chylack LT Jr, Wolfe JK, Singer DM, et al. The Lens Opacities Classification System III. The Longitudinal Study of Cataract Study Group. Arch Ophthalmol 1993;111:831-6.

16. Wei Q, Zhang T, Jiang R, et al. Vitreous Fibronectin and Fibrinogen Expression Increased in Eyes With Proliferative Diabetic Retinopathy After Intravitreal Anti-VEGF Therapy. Invest Ophthalmol Vis Sci 2017;58:5783-91.

17. Smeyne M, Smeyne RJ. Glutathione metabolism and Parkinson's disease. Free Radic Biol Med 2013;62:13-25.

18. Acharya S, Rodriguez IR, Moreira EF, et al. SPACR, a novel interphotoreceptor matrix glycoprotein in human retina that interacts with hyaluronan. J Biol Chem 1998;273:31599-606.

19. Chen M, Wu A, Zhang L, et al. The increasing prevalence of myopia and high myopia among high school students in Fenghua city, eastern China: a 15-year population-based survey. BMC Ophthalmol 2018;18:159.

20. Beuckmann CT, Gordon WC, Kanaoka Y, et al. Lipocalin-type prostaglandin D synthase (beta-trace) is located in pigment epithelial cells of rat retina and accumulates within interphotoreceptor matrix. J Neurosci 1996;16:6119-24.

21. Kristensen DB, Imamura K, Miyamoto Y, et al. Mass spectrometric approaches for the characterization of proteins on a hybrid quadrupole time-of-flight (Q-TOF) mass spectrometer. Electrophoresis 2000;21:430-9.

22. Lögdberg L, Wester L. Immunocalins: a lipocalin subfamily that modulates immune and inflammatory responses. Biochim Biophys Acta 2000;1482:284-97.

23. Taniguchi H, Mohri I, Okabe-Arahori H, et al. Prostaglandin D2 protects neonatal mouse brain from hypoxic ischemic injury. J Neurosci 2007;27:4303-12.

24. Jaggi GP, Flammer J, Huber AR, et al. Lipocalin-like prostaglandin D synthase in subretinal fluid of detached retinas in humans. Retina 2008;28:858-63.

25. Kuo HK, Chen YH, Huang F, et al. The upregulation of zinc finger protein 670 and prostaglandin D2 synthase in proliferative vitreoretinopathy. Graefes Arch Clin Exp Ophthalmol 2016;254:205-13.

26. Loukovaara S, Nurkkala H, Tamene F, et al. Quantitative 
Proteomics Analysis of Vitreous Humor from Diabetic Retinopathy Patients. J Proteome Res 2015;14:5131-43.

27. Fukuhara A, Yamada M, Fujimori K, et al. Lipocalin-type prostaglandin D synthase protects against oxidative stressinduced neuronal cell death. Biochem J 2012;443:75-84.

28. Xiong W, MacColl Garfinkel AE, Li Y, et al. NRF2 promotes neuronal survival in neurodegeneration and acute nerve damage. J Clin Invest 2015;125:1433-45.

29. Ishii T. Close teamwork between $\mathrm{Nrf} 2$ and peroxiredoxins 1 and 6 for the regulation of prostaglandin D2 and E2

Cite this article as: Wei Q, Zhang T, Fan J, Jiang R, Chang Q, Hong J, Xu G. Pathological myopia-induced antioxidative proteins in the vitreous humor. Ann Transl Med 2020;8(5):193. doi: 10.21037/atm.2020.01.63 production in macrophages in acute inflammation. Free Radic Biol Med 2015;88:189-98.

30. Kim KH, Sadikot RT, Xiao L, et al. Nrf2 is essential for the expression of lipocalin-prostaglandin D synthase induced by prostaglandin D2. Free Radic Biol Med 2013;65:1134-42.

31. Esworthy RS, Chu FF, Paxton RJ, et al. Characterization and partial amino acid sequence of human plasma glutathione peroxidase. Arch Biochem Biophys 1991;286:330-6. 